ARTICLE HISTORY: Received: December 20, 2021 Accepted: February 12, 2022 Published: February 19, 2022

\title{
A CROSS-CULTURAL AND INTERLANGUAGE STUDY OF REQUESTS IN THE KAZAKH AND ENGLISH LANGUAGES
}

\author{
Yerkebulan Zhumatayev \\ University of Szeged \\ Doctoral Institute, Doctoral School of Linguistics
}

\begin{abstract}
This paper provides some (more) insights into cross-cultural variation in speech act realization by analyzing English, Kazakh, Chinese and other languages requests. It aims to shows that the relationship between indirectness and politeness is interpreted differently across cultures.
\end{abstract}

Keywords: requests, WDCT, directness. indirectness, Politeness, East-West divide

One of the actual themes in the field of request is comparison DCT and naturally occurring requests. As Maria Economidou-Kogetsidis (Maria Economidou-Kogetsidis, 2013) investigates since the first extensive and systematic use of the written discourse completion test (henceforth WDCT) in the Cross- Cultural speech act realization projects (CCSARP) (Blum-Kulka, 1982; Blum-Kulka et al., 1989a), the DCT (written and oral) has been used to gather and compare data in the majority of empirical studies in pragmatics research (e.g. Blum- Kulka and Olshtain, 1984, 1986; Fearch and Kasper, 1989; Van Mulken, 1996; Lee, 2005; Woodfield, 2006; Bataineh and Bataineh, 2006; Dalmau and Gotor, 2007; Economidou-Kogetsidis, 2008, 2009; Woodfield and Economidou-Kogetsidis, 2010, to mention but a few). This has been primarily because of the administrative advantages of DCTs (especially WDCTs) which enable researchers to easily collect a large amount of data while controlling the social/situational variables involved in each situation tested. Yet, despite its widespread use, the WDCT has also been the most criticized elicitation instrument in cross-cultural and interlanguage pragmatics with the main criticisms being related to the WDCT's inability to capture the features of spoken language and natural interaction (for a full discussion see section 2.2). These criticisms and limitations have led many researchers to turn to the use of oral role-plays (Hassall, 1997, 2001, 2003, 2012; Goy et al., 2012; Felix-Brasdefer, 2007b; Otcu and Zeyrek, 2008; Economidou-Kogetsidis, 2012; Woodfield, 2012), which, if administered with care, have been found to yield data which approximate natural discourse (Kasper, 2000). If we use natural data it is impossible to use social characteristics of the informants (e.g. age, ethnic group, socioeconomic status, gender) because of being unreported and/or unknown. Another characteristic has DCT data. It requires students to read a written description of a social situation and asks them to write what they would say in that situation and more related to simulation. Apart from author pays attention to the difference between WDCT and DCT. Another issue has been the length of the situational prompt and the amount of contextual information that should be provided while using DCT because pilot-test result showed that participants need enriched external modification and elaboration than previous types of the most DCT studies to date have used. Next thing is difference between DCT and oral, interactive role-plays. The author gave Rintell and Mitchell's (1989) examples which showed that using DCT can be direct, using less polite language, while in a face to face encounter with another person. . some subjects are less comfortable using such direct language. If we want to examine the role of gratitude by native and non-native speakers of English, the best way is using WDCTs, oral DCTs, oral role-plays and naturally occurring examples. Also as author indicates that WDCT responses are shorter and contain a smaller range of acts, while DCTs seem to be appropriate instruments for discovering what semantic formulas are frequently used (or expected) in performance of a speech act.

As a data was chosen the current investigation from the social situation used involved an airline's call centre where the caller (speaker) rings in order to make a flight reservation or a 'standard' situation. The subjects of investigation were British English native speaker students of Anglo-Saxon origin. The participants of the investigation used: the most direct strategies (bald-on-record strategies), conventionally indirect strategies, non-conventionally indirect strategies (Hints). At the end the test results showed such characteristics:

- WDCT requests were found to be more direct compared to natural requests; - Natural requests included a significantly higher number of direct questions, WDCT requests included primarily want statements through the use of the 'I would like/I'd like' structure.

- It was found that the naturally occurring requests included less syntactic modification than the WDCT requests.

- While in natural data there was a statistical preference for hearer perspective, within the WDCT data there was a preference for speaker perspective.

Kazakh language is related to the Turkish language family among Azerbaijani, Turkmen, Qashqai, Gagauz, and Balkan Gagauz Turkish. Kazakhstan is situated in the central Asia. And if we want to study the correlation between English and Kazakh language requests, we should know about East-West divide in pragmatics. As requests divided into East-West the next paper reports an empirical investigation of Chinese requests using Hill et al's (1986) methodology. The study has yielded the following findings. (1) Chinese requests seem to be determined by Brown and Levinson's 
notions of power and distance, as are Japanese and American English requests as reported in Hill et al., (2) Chinese requests may not be as direct as has been argued in the literature. As the previous aims were: the first is to see if Chinese requests are similar to or different from Japanese requests and American English requests as reported in Hill et al. (1986), which will yield evidence for or against Brown and Levinson's (1987) theory of politeness. Secondly, to find out if Chinese requests are indeed as direct as has been widely believed in the literature. According to the investigation, first, the Chinese respondents used a smaller number of request expressions than their American counterparts. Secondly, while American English seems to favor one particular expression---May I borrow your pen?---among the most careful expressions, Chinese subjects appear not to have a clear favorite. The third difference one observes comparing, which indicates that respondents seldom if ever use "careful" request expressions with "uninhibited" categories of people. One similarity: all of the most frequently used expressions of the Chinese respondents also include Can in the head act (Blum-Kulka and Olshtain, 1984), which has more than one form in Chinese (能 neng, 可以 keyi). They differ only in the moves that occur before the head act (e.g. I'm sorry for interrupting. ... Do you have a pen. . .. I don't have a pen. . . My pen is broken. ...).

This study has yielded similarities as well as differences. The authors have different meaning about Japanese politeness explaining that since Western politeness is seen as a means to avoid imposition and the apparent imposing expression "yoroshiku onegaishimasu» is used as a politeness token in Japanese, Japanese politeness has to be different from Western politeness. There has been evidence that negative face is just as valid in Japanese as it is in Western cultures---that Japanese speakers are found to use euphemisms, hedging, questioning, and apologizing to signal their respect for the hearer's territory and that negative face considerations "constrain the use of desiderative, emotive/affective terms, the expression of the speaker's intentions, or questions on the hearer's skills and abilities" (Pizziconi, 2003:1479). Even Gu (1990) and Mao (1994) explained Chinese politeness by four maxims. If Chinese requests are just as sensitive to Brown and Levinson's P, D, and R and just as indirect as requests in Western cultures, they once again find evidence that East and West may not be as different as believed by those holding the Different Position in the East-West debate. It was found that Chinese are more similar to Americans than they are to the Japanese--that they, too, perceive less difference in the degree of politeness. At the end it was clarified that: First, Chinese requests are found to be similar to American and Japanese requests. Second, Chinese speakers, as do Japanese and American speakers, request according to their judgment of Brown and Levinson's notions of power and distance. Third, the data in this study does not support the claim that Chinese requests are typically direct. On the contrary, they are found to be just as indirect as American English and indirection is rather closely related to speaker's effort to mitigate the face threat of the act of requesting.

Our aim is to study kazakh students' requests during a stay abroad. And the next theme is connected with it. As the authors wrote measuring pragmatic competence is a methodologically difficult endeavour, but is of great importance to contexts like stays abroad, which are conducive to changes in pragmatic abilities. It was proposed a mixed-method approach to this issue, using functional speech act analysis alongside a newly developed method for lexical analysis. Both methods are used to evaluate the development of pragmatic competence in German high-school students before and after a 10-month stay in Canada, with corresponding Canadian native speaker data for direct comparison. Authors agree that a reliable assessment of learner performance can only be achieved by either comparison with parallel native speaker data or by testing learner performance in large-scale perception studies involving a sizeable number of native speakers. The two levels of abstraction considered here are henceforth referred to as the functional level (analyses focus on the strategies speakers select to achieve communicative goals) and the formal level the formal analysis employed in this paper considers the lemmas used for realizing the request in question, and does not take syntactic patterns into account), the latter in this case instantiated by the lexical material used in a given utterance. Before authors put these two questions:

1) Can the occurrence and frequency of functional features be utilized as an indicator of pragmatic competence? In other words, do the functional features found in the learner utterances before and after the SA show any convergence towards the native speaker utterances, suggesting an increase in pragmatic competence?

2. In a similar fashion, is the lexical material used by the NNS for realizing requests more similar to the native speaker requests after the SA than before, indicating gains in pragmatic competence? And as data collection instrument was used the Questionnaire on English Usage (QEU), a written mixed-task multi-focus questionnaire which has been used to compare a number of first, second and foreign language varieties of English such as American, Canadian, English, Irish and Namibian English, because the QEU elicits a range of interactional and non-interactional pragmatic phenomena, including requests. The scenario used for that study was a Written Discourse Completion Task (WDCT). They have compared two methods for measuring pragmatic competence by applying them to the case of a sojourn abroad, comparing the requests produced by German high-school students before and after their SA with a com-parable NS group. The results show that both methods have particular strengths for their individual applications relative to the research question and focus of the data analysis.

When we do request, how do we use it? Which one direct or indirect requests? Nicolas Ruytenbeek studied this theme, especially: 
- assumes that there are situations where the conventionalized IR (1) and the imperative (2) are two plausible alternative ways to perform one;

- argue that, for standard RT, the "request" assumptions communicated by conventionalized Can you VP?

- underlines why, according to RT, extra inferential steps are predicted for conventionalized IRs.

- argues that, all other things being equal, in a situation where a speaker performs the act of requesting, both an imperative stimulus and a conventionalized IR expression can achieve maximal relevance because the latter do not increase the processing costs relative to imperatives.

In the relevance-theoretic framework, maximal relevance is achieved when processing effort is minimized and cognitive effects are maximized. In line with the presumption of optimal relevance, the addressee (A) of an utterance not only assumes that the speaker (S)'s utterance is relevant enough for it to be worth processing: he expects $\mathrm{S}$ to choose an utterance that is the most relevant one compatible with her preferences and her abilities. A detailed analysis of the request forms indicates that the French equivalent of conventionalized Could you VP? (Pourriez-vous VP?) requests were no more frequent in the higher-status condition than in the equal-status condition. Instead of increasing the politeness of their message with Could you VP? when they addressed a higher-status person, the participants used specific linguistic devices, such as formal greetings and polite V-forms of address. If ability interrogatives Can you VP? are frequently used in the performance of requests in British and American English conversations, this preference is best accounted for in terms of speakers' desire to avoid being perceived as impolite. As it's said recent experimental evidence indicated that, in some situations, this extra inferential work does not translate into extra processing costs, which points to the facilitative role that pragmatic routines play in IRs understanding. This implies that both Can you VP? and imperative stimuli can achieve maximal relevance in the performance of requests. While politeness effects can, in theory, be triggered by conventionalized indirect expressions, the sort of politeness associated with these expressions generally goes unnoticed, and it is, therefore, unlikely that they communicate politeness as an extra cognitive effect. However, IRs requests differ from imperative requests in the sense that the former match speakers' preferences more than imperatives do: they enable them to avoid being perceived as impolite. As a consequence, unlike the imperative sentence-type, uttering the conventionalized Can you VP? can be optimally relevant for the performance of requests in many situations.

The requests in kazakh language are used differently, especially they are connected with literature. And one who studied this theme in our days is Asylbek Meyrbekov (2018). His Phd. thesis work «Teaching requests in kazakh language using communicative aspects» is gives more information.

The results of the present study show that in all examined languages requests can be realized at different levels of directness and their illocutionary force can be downgraded by means of internal (syntactic and lexical) and external modification. Cross-cultural differences have been mainly established at the level of substrategies, their linguistic realizations, restrictions on their applicability and, consequently, the frequencies with which they were used.

\section{References:}

1.Maria Economidou-Kogetsidis, 2013. Strategies, modification and perspective in native speakers' requests: A comparison of WDCT and naturally occurring requests. Journal of Pragmatics 53, 21-38.

2. Rong Chen, Lin He, Chunmei Hu, 2013. Chinese requests: In comparison to American and Japanese requests and with reference to the "East-West divide". Journal of Pragmatics 55, 140-161.

3. Friederike Sell, Katrin Renkwitz, Pawel Sickinger, Klaus P. Schneider, 2019. Measuring pragmatic competence on the functional and lexical level: The development of German high-school students' requests during a stay abroad in Canada. Journal of Pragmatics 146, 106-120.

4. Nicolas Ruytenbeek, 2019. Indirect requests, relevance, and politeness. Journal of Pragmatics 142, 78-79.

5. Asylbek Meyrbekov, 2018. Teaching requests in kazakh language using communicative aspects. Phd. Thesis work, 50-51. 